\title{
Industria Farmacéutica y Psiquiatría
}

RESUMEN: Se analiza el cambio que ha experimentado la industria farmacéutica desde una labor centrada en la investigación a una actividad volcada en el marketing y la promoción. Describimos las diferentes estrategias que están utilizando para ello y discutimos las repercusiones que están teniendo sobre la psiquiatría (en la investigación, en el modelo de enfermedad mental, en los psiquiatras, en la asistencia y en el discurso social)

Palabras clave: Industria farmacéutica, psiquiatría, relación, consecuencias.
SUMMARY: We analyse the shift of the pharmaceutical companies activity: from investigation to marketing and promotion. We describe the different strategies that they use for this purpose and we discuss about the consequencies on psychiatry (on investigation, on mental disorder model, on psychiatrists, on mental health attention and on social speech).

Key words: Pharmaceutical industry, psychiatry, relationship, consequences.

Siempre me esfuerzo por agradar y logro que mis beneficios pasen inadvertidos. He aplicado el mismo principio en la publicidad. Nunca le pido a la gente que compre. Rara vez doy precios. Ofrezco servicios, tal vez una muestra gratis. Suena altruista. No son cosas que hace la gente egoísta... Luego, como reacción natural, se esfuerzan en encontrar la forma de devolver la cortesía, comprando.»

C .Hopkins (padre de la publicidad moderna) 


\section{Introducción}

Como señala Healy (1), con frecuencia los estudios sobre la historia y el desarrollo de la psicofarmacología moderna se limitan a relatar una cronología de fechas, descubrimientos e investigadores sin analizar, o relegando a un papel secundario, otro tipo de realidades tales como los factores sociales, culturales, políticos y económicos, como por ejemplo la influencia de los intereses comerciales de la industria farmacéutica. Para bien o para mal, desde la aparición de la clorpromacina a finales de los años cincuenta, el descubrimiento, investigación y difusión de los fármacos activos sobre el sistema nervioso central no ha sido tanto una obra de científicos académicos o médicos de hospital como de ejecutivos y científicos de las empresas farmacéuticas (2).

La industria farmacéutica se define a sí misma (Sachs y Dieck, 1989) (3) como «una gran corporación, abierta al conocimiento público, que depende de la investigación y desarrollo para poder crecer y tener beneficios». En nuestra opinión, si bien en el campo de los psicofármacos podríamos aceptar que se aplicara esta definición a las fases iniciales de su desarrollo hace cuarenta años, actualmente los cambios que se han producido en la propia industria y en el contexto político, económico y social no la sostienen. Para muchos, actualmente la industria (psico)farmacológica se parece más a una gran corporación, de difícil acceso al conocimiento público, que depende fundamentalmente de la promoción o el marketing, y en menor medida de la investigación, para poder crecer y ganar cada vez más y más beneficios.
Este papel central de la industria farmacéutica en la investigación no deja de ser controvertido. Basándose en evidencias científicas o comerciales, la realidad es que en las últimas décadas la mayoría de las personas con problemas de salud mental, independientemente de su gravedad, diagnóstico o nivel sanitario en el que son atendidos son tratados con psicofármacos. Desde la industria este hecho es muy celebrado. Señalan que sin su contribución no se hubiera logrado el desarrollo de un arsenal terapéutico «eficaz» y no se hubiera avanzado en el conocimiento de las bases «biológicas» de las enfermedades mentales. Desde posiciones más críticas sin embargo, se cuestiona por un lado la sobreutilización de los psicofármacos y sus consecuencias y, por otro, cómo los intereses comerciales están determinando la calidad de los ensayos clínicos, las líneas de investigación en psiquiatría y salud mental, la categorización de enfermedades y la nosología, el modelo de enfermedad mental, las prácticas asistenciales y la prescripción de los profesionales. Este artículo tiene como objetivo señalar estos aspectos controvertidos para contribuir al debate existente entre los intereses de la industria farmacéutica y la medicina, en concreto con la psiquiatría.

La transformación de la industria: de la investigación a la promoción

Los inicios de la psicofarmacología moderna coincidieron en los países industriales occidentales en los años cincuenta y sesenta con las políticas de bienestar, que en el campo de la salud mental plantearon 
la necesidad de una reforma de la atención psiquiátrica de manera que el eje de la atención pasara de las instituciones manicomiales a una atención comunitaria. Aunque el poder tener un tratamiento farmacológico que controlara los síntomas de los pacientes podría ayudar a este objetivo, en esos momentos de pleno auge de las corrientes sociales en psiquiatría y con un clima de contestación frente a las instituciones de control social no parecía adecuado abrir este mercado. Sin embargo, la colaboración de químicos de estas industrias con clínicos próximos a ellas llevaron, no sin una dosis de intuición y tesón, a probar distintas moléculas en enfermos mentales graves. Los resultados eran esperanzadores y levantaron expectativas en los ejecutivos de las empresas y en la propia profesión médica. Se ha señalado que la rápida introducción de la clorpromacina (en aquella época los requisitos de la administración no estaban tan regulados como actualmente) ayudaría considerablemente al proceso de desinstitucionalización al conseguir mejorar los síntomas y controlar las conductas de los enfermos mentales más graves. Además abrió unas enormes expectativas sobre la investigación de mecanismos bioquímicos cerebrales de las enfermedades mentales. En poco tiempo se pasó de una situación en que los tratamientos farmacológicos eran considerados como accesorios a una nueva en que constituirían la parte central del tratamiento y el estímulo para entender la «naturaleza fundamental» de los trastornos mentales. Esto se denominó la revolución psicofarmacológica (4).
Sin embargo, la puesta en practica de las reformas psiquiátricas, la expansión de la industria farmacéutica y la primacía del paradigma biológico en psiquiatría coincide con la crisis del estado de bienestar en los años setenta y el desarrollo de una serie de cambios político-económicos y socioculturales que han facilitado el negocio de la industria farmacéutica. Por un lado, la revolución neoliberal encabezada por el gobierno de Ronald Reagan y secundada posteriormente en Europa por el de Margaret Thatcher, se propuso convertir también el terreno del cuidado de la salud en un nuevo campo de mercado (5). Las limitaciones del Estado de Bienestar a partir de entonces y el permiso de entrada de capitales privados a los sistemas sanitarios amenaza con acabar convirtiendo la salud en un producto de consumo y no tanto en un derecho garantizado por el Estado. A través de aseguradoras (que multiplican su negocio en este nuevo contexto) se pueden consumir ecografías, análisis, chequeos que aunque no estén fundamentados, el paciente solicita. En los sucesivos años, los sistemas nacionales de salud europeos podrían quedar relegados para los que no pueden o no quieren pagarse una mutua, para las enfermedades caras que no resultan rentables (cirugías mayores, enfermedades crónicas, atención a los mayores...) y, esto es importante, para sufragar el gasto farmacéutico de todos.

En este nuevo contexto determinado por las políticas neoliberales donde el mercado se constituye como el principal regulador de las relaciones sociales, el individualismo aparece como el valor hegemónico. El malestar cultural y social proyec- 
tado en el individuo también se mercantiliza. La rutina es sustituida por la inestabilidad y el riesgo como campo semántico que caracteriza la cultura y las relaciones sociales, a la vez que se ensalza el éxito individual. La frustración que genera esta contradicción provoca diversas formas de sufrimiento, que la industria puede contribuir a medicalizar de diferentes maneras, para abrir nuevos mercados a los ya existentes $(6,7)$.

En unas décadas la industria farmacéutica pasa a ser una de las actividades más lucrativas, con crecimientos medios de beneficios (en términos de retorno de inversión) superiores al $15 \%$ durante la pasada década, que contrastan con el 5\% de media del resto de las industrias que aparecen en el listado anual de 500 industrias que elabora la revista Fortune (8). Las ventas de las compañías farmacéuticas norteamericanas en todo el mundo crecieron de los 22.000 millones de dólares en 1980 a los 181.800 millones de dólares en el año 2001 (9); las ventas totales en el año 2001 (9) fueron de 364.200 millones de dólares (el $88 \%$ en EEUU, Europa y Japón) ${ }^{1}$ y los psicofármacos en particular se sitúan entre los más rentables (tablas I y II).

Estas formidables ganancias ${ }^{2}$ se explican en parte por la potenciación de los departamentos de marketing y el cambio de estrategia de la investigación que pasa a depender menos de los aspectos clínicos como de los aspectos de mercado (10). El crecimiento de los costes de desarrollo de una nueva medicina (desde los 54 millones de dólares en 1976 hasta los mas de 500 a finales en el año 2000) ${ }^{3}$, que justifica para la industria los elevados precios y los derechos sobre las patentes, depende más del crecimiento de los costes de marketing que los de investigación. En el año 2000, las principales industrias farmacéuticas destinaron un $12 \%$ del coste a investigación y desarrollo frente a un 30\% destinado al marketing $(11,12)$; se calcula que, como promedio, en una compañía farmacéutica el $22 \%$ de los empleados se dedican a la investigación frente al 39\% dedicados al marketing; según el National Institute of Health Care Management (13) los gastos en promoción ascendieron de 9.000 millones de dólares en 1996 a 15.700 millones en el año 2000 en Estados Unidos. $^{4}$

En definitiva, hace cuarenta años la aparición de las nuevas medicaciones inició la llamada revolución psicofarmacológica de la psiquiatría. El interés de la industria por el beneficio sin límites, su orientación hacia la promoción, y su enorme poder de influencia ha dejado aquella revolución en un simple motín cuyas expectativas científicas no se han cumplido. La verdadera revolución ha sido el desarrollo en las últimas décadas de una psiquiatría al servicio del mercado: una psiquiatría no biológica, sino comercial.

\section{EstRATEGIAS}

Es razonable pensar que en un mercado donde se invierte tres veces más en marketing que en producción, expondrá a los médicos, instituciones y sociedades científicas, agencias estatales, asociaciones de familiares y pacientes, etc. a fuertes presiones y variadas estrategias de ven$\mathrm{ta}^{5}$. Cuando un «product manager» trata 
de «posicionar» un producto en un sector tiene que trazar una estrategia competitiva en la que ha de evaluar las cinco fuerzas que impulsan la competencia y a las que hay que enfrentarse:

- Poder de negociación de los proveedores: la provisión de materia prima (nuevas moléculas), que en el caso de otros productos puede ser determinante por su escasez o coste, no era un problema para la industria farmacéutica hasta ahora.

- Poder de negociación de los clientes: como el mercado de los fármacos está regulado, antes de negociar con los clientes hay que conseguir la aprobación del producto y negociar previamente con organismos como la FDA, la Agencia Europea del Medicamento o las agencias de cada país. Después, quien compra básicamente en un Sistema Nacional de Salud es el Estado, aunque para productos que no necesitan receta, los pacientes también pueden ser clientes directos. La industria farmacéutica tiene que negociar con el Ministerio de Sanidad que debe decidir qué psicofármacos aprueba, para qué indicaciones y a qué precio. En esta negociación de alto nivel entran en juego las contraprestaciones que puede realizar la industria farmacéutica si sus productos son aprobados: desde seguir invirtiendo en el país hasta encargarse de la docencia de los médicos... Por último, los clientes inmediatos son los prescriptores de los productos, es decir, los psiquiatras y resto de médicos. Teóricamente los médicos no tienen un poder de nego- ciación real sino que tienen que prescribir los psicofármacos más adecuados, pero son persuadidos por las compañías de que sus productos son los mejores.

- Amenazas de productos o servicios sustitutivos: la amenaza a los psicofármacos la podrían constituir los abordajes de la enfermedad mental alternativos (psicoterapia, rehabilitación psicosocial, psicoeducación...) Aunque estos abordajes no son incompatibles con la farmacoterapia, sino que, al contrario, sus efectos se pueden potenciar, es importante para la industria farmacéutica mantener un modelo biomédico de la enfermedad mental para no ceder terreno y que sus productos sean lo imprescindible de los tratamientos (los otros abordajes también son o deberían ser imprescindibles).

- Riesgo de entrada de nuevas empresas: tras las últimas fusiones el control del mercado lo ostentan unas pocas megamultinacionales y el riesgo de que nuevas empresas les quiten su cuota de mercado parece lejano (las nuevas tecnologías basadas en ingeniería genética podrían revolucionar el mercado) (8). Sin embargo, la aparición de los genéricos comercializados por laboratorios más pequeños con un precio inferior al principio activo de marca son una dura competencia cuando caduca la licencia de la patente del producto. Hay tres estrategias básicas para luchar contra esta fuerza: 1) mientras dura la licencia, comercializar el producto al precio más alto posible 
para poder rentabilizarlo y cuando aparezca el genérico bajar el precio al de referencia para seguir siendo competitivo. 2) Patentar distintas presentaciones en distintos tiempos para que cuando caduque la licencia de las cápsulas por ejemplo, quede todavía la de los comprimidos, la solución, el inyectable o la forma de liberación retardada. 3) Cuando va a caducar la licencia, se puede cambiar ligeramente la molécula (poner o quitar algún radical p. ej. y vender que este «nuevo» producto es mucho mejor que el anterior) para competir con los genéricos de la molécula antigua.

- Rivalidad entre empresas actuales: esta es una fuerza importante porque muchas veces el mismo psicofármaco se comercializa con distintas marcas y muchos psicofármacos son semejantes entre si. Por ello, los artículos científicos publicados en las revistas tienden a hacer una especie de «publicidad agresiva» realizando ensayos que denostan al producto rival.

La tarea de enfrentarse a las 5 fuerzas que impulsan la competencia precisa de estrategias más específicas que garanticen las ventas del producto. La mayoría de ellas nos incluyen a los psiquiatras y médicos generales como principales propagandistas de los psicofármacos con nuestras prescripciones. No cabe pensar entonces que los médicos sean unas víctimas de la manipulación de la todopoderosa industria farmacéutica sino más bien unos cómplices conscientes o inconscientes, que participan de sus estrategias de mercado.

\section{Estrategia sobre la investigación}

Desde finales de los años setenta la inversión pública en investigación biomédica no se ha desarrollado adecuadamente, lo que ha permitido que la industria tenga un mayor papel en su financiación y realización. La industria argumenta que su contribución a la financiación de la I+D total es del $70 \%(14,15)$ pero no hay que olvidar que este dinero que aporta la industria ha sido fruto de un acuerdo entre las empresas y el estado, que a cambio de seguirles permitiendo unos amplios márgenes de beneficios les obliga en reciprocidad a invertir en I+D y en programas de formación ${ }^{6}$. El problema es que como este dinero no esta regulado de forma adecuada ni democrática, termina sirviendo a los propios intereses de mercado, es como una reinversión. La industria dice que gasta mucho en $\mathrm{I}+\mathrm{D}$, pero una parte sustancial de esos recursos se dedica a pseudoensayos clínicos, que no son en realidad más que campañas de marketing $\mathrm{y}$, como veremos, gran parte de la formación continuada que realiza con los médicos se basa en la promoción de sus productos directa o indirectamente (16).

Parece demostrado que la investigación en manos de la industria tiene un sesgo sistemático que la favorece ${ }^{7}$. Por ejemplo, en un meta-análisis sobre la eficacia de los antidepresivos (17) (Freemantle et al., 2000) el predictor más consistente de la eficacia del antidepresivo en un ensayo era si estaba patrocinado por la empresa fabricante. Esto parece consecuencia de la orientación, anteriormente señalada, de la investigación hacia el marketing. En los últimos años, han proliferado consultoras 
que se encargan de diseñar, realizar, informar e incluso presentar los resultados para solicitar la aprobación final de nuevos fármacos. Tradicionalmente, los ensayos clínicos eran proyectados, llevados a la práctica e interpretados por investigadores académicos independientes, pero las empresas han ido sustituyéndolos por las llamadas 'organizaciones de investigación bajo contrato' (CRO por sus siglas en inglés), que son grupos de investigadores privados, sin lazos con las universidades y los organismos públicos de investigación que al final son los que controlan el proceso(10).

La investigación está orientada desde el principio por tanto a pasar los controles de las agencias reguladoras como la FDA y a buscar un espacio en un mercado cada vez más competitivo. Esto tergiversa por completo los ensayos clínicos, que son diseñados desde el principio para favorecer a sus productos. Se pueden poner muchísimos ejemplos de sesgos en la investigación de los psicofármacos $(18,19)$,desde cómo se seleccionan los pacientes, los criterios clínicos, los tamaños muestrales, las escalas utilizadas, la duración de los estudios, el registro de efectos secundarios... Un ejemplo ilustrativo lo tenemos en los ensayos clínicos de los nuevos antipsicóticos (19), en los que es frecuente que se utilicen dosis altas y fijas de haloperidol (entre 10 y $20 \mathrm{mg} /$ día) en el grupo control frente a dosis variables y relativamente bajas de los nuevos antipsicóticos (luego en el postmarketing ya se encargaron de decirnos que se necesitaban dosis más altas). Esto sobredimensiona tanto la eficacia como la seguridad y tolerancia de estos fármacos. Otro ejemplo de cómo los ensayos se diseñan interesadamente lo tenemos con los llamados fármacos «me too» (yo también). En la pasada década el éxito de ventas de los primeros ISRS inundaron el mercado de nuevas moléculas «me too» que se lanzaban con el mensaje de ser al menos tan eficaz como la imipramina u otro de los ISRS basándose múltiples estudios de tamaños muestrales de baja potencia estadística que, lógicamente, no hallaban diferencias significativas con el fármaco control. Ahora parece que estamos asistiendo a un nuevo fenómeno de antipsicóticos caros «me too».

\section{Estrategias sobre la información médica}

\subsection{Revistas}

Las revistas médicas no deben ser meros órganos de difusión de informaciones sanitarias, sino que constituyen la pieza fundamental del proceso de evaluación y control de calidad de cualquier investigación. La preocupación existente en la revistas más prestigiosas por la influencia de los intereses económicos en la calidad de la información científica de los artículos les llevó en Septiembre de 2001, a realizar un editorial conjunto denunciando esta situación y a plantearse tomar una serie de medidas para modificar coordinadamente las condiciones para aceptar un trabajo en sus páginas (20-22). Según los cálculos del Hospital General de Massachussets (20), entre el 30\% y el 50\% de los contratos de investigación que le son propuestos por la industria farmacéutica para desarrollar en su hospital incluyen «cláusulas de publicación inaceptables» ${ }^{8}$. 
Existen múltiples formas en las que la industria controla la información de lo que se publica. En primer lugar tenemos las presiones directas sobre los consejos editoriales. Las reglas del Comité Internacional de Editores de Publicaciones Médicas (22) establecen que quienes toman importantes decisiones en publicaciones de este tipo no deben tener intereses personales, profesionales o financieros en algunos de los temas que tratan. Según el Lancet (22), uno de los editores del British Journal of Psychiatry, vinculado a un centro de formación de una compañía farmacéutica de la que recibía 2000 dólares anuales, ha sido recientemente cuestionado por incluir artículos en los que se favorecían a los productos de dicha compañía. En las revistas pequeñas y de ámbito más nacional es frecuente ver en los consejos editoriales los mismos nombres que dirigen investigaciones financiadas por la industria y que dan los seminarios que patrocinan el producto. Estas revistas no tienen problemas de financiación. En cambio, en nuestro país hace dos años la revista Psiquiatría Pública desapareció por falta de financiación tras la retirada masiva de publicidad de las compañías farmacéuticas a raíz de un editorial (23) que no gustó a la industria y sus colaboradores (financiar anualmente esta revista no costaba más que el gasto de media docena de psiquiatras en un congreso de la APA).

En segundo lugar, como hemos señalado antes, los departamentos de marketing no sólo controlan los diseños de las investigaciones, sino que dirigen y supervisan la redacción de los artículos, introduciendo sesgos o interpretaciones que favorecen al producto (19). En ocasiones los artículos son firmados por autores de prestigio que no habían participado directamente en el análisis de los datos $(22,24)$ o se publica material no revisado en un suplemento de una prestigiosa publicación.

Otro tipo de control sobre la información tiene que ver con el sesgo de publicación (25) por el cual los estudios de investigación con resultados «positivos» estadísticamente significativos se publican en mayor medida que los que obtienen resultados «nulos» o «negativos». La consecuencia de este sesgo es que la información de dominio público de las revistas científicas no es representativa de la totalidad de la evidencia investigadora porque los estudios muestran resultados en una sola dirección. Otro sesgo, en el que la industria tiene un papel destacado, es el de publicación múltiple o «salami slicing», que consiste en publicar un solo estudio con resultados positivos de forma troceada en distintos artículos. Por ejemplo, si es un multicéntrico, se publican los resultados de cada centro de investigación en cada artículo, o si se trata de un estudio prospectivo, se sacan diferentes artículos en distintos momentos del estudio o simplemente se van publicando partes de los resultados. Es importante para ello, ir alterando el orden y la composición de los autores para que parezcan estudios distintos ${ }^{9}$. El problema central de esta influencia de los intereses económicos es que amenaza la imparcialidad y objetividad de las publicaciones médicas.

2.2 Guías de clínicas de consenso Las guías clínicas pueden ser un po- 
deroso instrumento para determinar las prácticas de los médicos. Recientemente un estudio publicado en JAMA (26) en el que se analizan las relaciones entre los autores de las guías clínicas y la industria farmacéutica concluía que la mayoría de ellos recibían dinero de la industria o trabajaban como asesores para ella. Sin embargo los autores no declaraban en general este tipo de conflictos de intereses en la publicación. Las guías clínicas analizadas cubrían los campos donde más había crecido el gasto y la prescripción. Igualmente se han analizado las conferencias de consenso (25). En psiquiatría podemos ver, por ejemplo, cómo la guía de tratamiento de la esquizofrenia editada por la APA está patrocinada por los principales laboratorios que han puesto a la venta los últimos antipsicóticos del mercado (19).

2.3. La promoción directa al psiquiatra.

Los médicos recibimos continuamente información por parte de los laboratorios que invierten mucho dinero en ella. Para algunos es la principal fuente de actualización farmacológica y clínica. Esta información no puede considerarse científica, sino que tiene objetivos comerciales bien estudiados tanto en el contenido como en la presentación. Como señalan Sheldon y Smith (27) a las tradicionales formas de promoción (anuncios en revistas, envíos por correo e información de los representantes) se han añadido formas indirectas de promoción como publicaciones, conferencias seminarios que analizaremos luego. Los médicos generalmente niegan que la publicidad de las compañías influya en sus decisiones terapéuticas (28) aunque la mayoría de las evidencias demuestran lo contrario (29). El contacto regular con los representantes de los laboratorios se relaciona con un incremento de la prescripción de sus productos (30); los médicos expuestos a la publicidad son más propensos a aceptar más las evidencias comerciales que las científicas (31) y la publicidad de los laboratorios se asocia con la dificultad de muchos médicos para valorar adecuadamente la literatura y con una mayor tendencia a una prescripción inadecuada (32). Si la publicidad no influyera en la prescripción los laboratorios no gastarían tanto dinero en hacerlo.

Otra estrategia es la promoción a través de pseudoensayos clínicos, remunerados por supuesto, y de escasa calidad metodológica, cuyo verdadero objetivo es inducir la prescripción de un nuevo producto o una nueva indicación de un producto existente.

\section{Control de los incentivos}

La publicidad y los incentivos a los médicos en forma de distintos tipos de regalos y ayudas económicas para viajes a congresos, etc. supone una parte sustancial de los presupuestos de la industria farmacéutica (33), (se calcula que el gasto medio por médico en Estados Unidos es de 8.000 dólares anuales) (34). Los conflictos éticos que se producen en relación a las ayudas económicas y regalos de la industria han sido desde hace tiempo una fuente de preocupación para la profesión médica y la sociedad. De nuevo los médicos minimizan el papel que estos regalos tienen en su praxis cuando desde las ciencias sociales está muy bien estudiado que 
esta es una de las claves del éxito en la comercialización de cualquier producto ya que al aceptar un obsequio, quien lo recibe queda de alguna manera sometido a una especie de deuda de gratitud, que asegura que la deuda terminará siendo pagada. Desde distintas instituciones y sociedades médicas se han intentado establecer códigos de conducta para los profesionales en los que se especifican cuáles son las ayudas económicas y los regalos que son éticamente aceptables y cuáles no $(35,36)$. $\mathrm{Si}$ uno analiza el panorama actual en la profesión psiquiátrica a la luz de las recomendaciones de estas asociaciones el panorama es éticamente desalentador.

Los laboratorios disponen de diversos métodos para intentar conocer cuál es la prescripción de un médico determinado y adaptar individualizadamente los incentivos a ese médico $(10,37)$. Por un lado cuentan una base de datos con la cantidad de recetas por marca y por médico, actualizada mensualmente, a partir de una sofisticada red de información en las farmacias. Si esta información no está disponible realizan encuestas a través de compañías comerciales que se presentan como empresas investigadoras en las que al médico se le da un pequeño obsequio. Esta información es muy valiosa para las compañías a la hora de clasificar a los profesionales según las características personales y según su patrón de prescripción y de fidelidad al producto y establecer los incentivos individualizadamente (38).

Se establece así una curiosa jerarquía entre los médicos que recuerda a la de las empresas dedicadas a las ventas de productos a domicilio. En lo más alto de la escala estarían los expertos de prestigio ligados muchas veces a la Universidad y que actúan como asesores de la industria (muchas veces no reconocido públicamente como se ha visto en el caso de las guías), cobrando mucho más dinero del que reciben de su sueldo público, seguidos de expertos locales y finalmente de los psiquiatras de los centros que a su vez inducen la prescripción del médico de atención primaria. Es la cultura del «tupper-ware» psicofarmacológico, un dispositivo comercial muy bien diseñado y frente al que las administraciones no reaccionan incentivando adecuadamente a sus profesionales.

\section{Estrategia sobre la formación de los psiquiatras}

La formación médica continuada (FMC) es una actividad que requiere gran cantidad de fondos. Aunque es difícil de calcular cuál es la contribución exacta, está claro que la industria farmacéutica tiene un papel destacado en la financiación de la FMC. Las sociedades científicas y las instituciones docentes de toda índole se ven abocadas a solicitar el apoyo de las empresas farmacéuticas para poder realizar sus actividades científicas, dado el poco apoyo que se recibe desde las administraciones públicas (16). Estas últimas, a través de los acuerdos con farmaindustria obligan a los laboratorios a destinar una parte de sus presupuestos a financiar actividades de FMC, a cambio de mantener un margen alto de beneficios. Al igual que en el caso de la investigación el problema es que este dinero, en cierto sentido de todos, no está adecuadamente regulado. Jun- 
to a actividades que no dejan duda de su calidad e independencia científica existen muchas otras en el que el interés comercial es el principal objetivo. Esta dependencia cada vez mayor de la industria farmacéutica y los conflictos de intereses que se producen son objeto de una preocupación creciente en algunas asociaciones médicas. La Asociación Médica Americana (AMA) ha publicado recientemente una serie de recomendaciones destinadas a regular las relaciones entre las sociedades médicas y la industria farmacéutica (38). Para la AMA, las sociedades científicas deberían evaluar cuidadosamente y de manera responsable los sesgos en los cursos, proyectos y políticas que llevan adelante con apoyo de la industria farmacéutica. La cuestión reside en si nuestras asociaciones científicas cumplen unos mínimos éticos en este conflicto de intereses.

Uno de los principales problemas en el campo de la psiquiatría que se deriva de que la administración deje una parte sustancial de la FMC en manos del apoyo financiero de la industria es el sesgo de contenidos. La mayor parte de esta inversión está destinada a actividades de FMC de contenido biológico o que traten de intervenciones farmacológicas, promoviendo de esta manera un modelo de enfermedad mental tremendamente reduccionista. En nuestro país, las sociedades científicas que se aproximan más a este modelo reciben importantes cantidades de fondos de la industria para sus congresos, publicaciones, cursos y divulgación mediática, mientras que aquellas asociaciones que integran otros aspectos cruciales para los enfermos como la rehabilitación psicosocial, la psi- coterapia o la problemática asistencial tienen serias dificultades en poder llevar a cabo sus proyectos por el escaso apoyo con el que cuentan.

No sólo existe un sesgo general de contenidos, sino que además muchas de las actividades destinadas a dar a conocer los nuevos fármacos o nuevas indicaciones a las que se invitan constantemente a los médicos presentan informaciones tremendamente sesgadas que favorecen al producto de la compañía patrocinadora (32). Este tipo de eventos en los que un profesional de prestigio bien pagado (de 2000 a 3000 dólares más gastos de acomodación por conferencia según The Guardian) (24) diserta sobre aspectos clínicos o terapéuticos introduciendo con mayor o menor sutileza mensajes sobre el producto en cuestión y que se acompañan de ágapes sociales desproporcionados son cada vez más frecuentes. Existen estudios que demuestran cómo la participación en estos eventos cambian la prescripción a favor del producto en cuestión, incluso cuando están organizados por una institución o sociedad independiente $(39,40)$.

\section{Estrategias sobre la información a la opinión pública}

El público, incluso muchos médicos, no se da cuenta hasta qué punto se usan los medios de comunicación para promocionar los productos de la industria (10). En líneas generales, la meta de los medios -vender diarios o ganar «ratings»- coincide con la de la industria y el cuidado de la salud es un buen mercado informativo. Las historias de logros importantísimos («break-through»), lanzados desde la in- 
dustria, que se repiten en los medios informativos hasta el cansancio, no sólo estimulan a los consumidores para que pregunten a sus doctores acerca de nuevos tratamientos, sino que muchos estudios han demostrado que éstos también influyen sobre los propios médicos.

Existen consultores de relaciones públicas especializados en el cuidado de la salud, que cubren la necesidad de «noticias» que tienen sus contactos mediáticos. El paquete clásico incluye la investigación que se da a conocer en una conferencia o en un día dedicado a tomar conciencia de una enfermedad (disease awareness day), un experto en medicina para agregar credibilidad, y un paciente que va a aportar el ángulo de interés humano. Otra estrategia popular es diseñar una investigación cuyos resultados puedan usarse para generar cobertura mediática. $\mathrm{O}$ traer «prominentes expertos extranjeros» para que den entrevistas en los medios junto con reuniones de profesionales de la salud.

La industria también ha apoyado muchos grupos de pacientes (que resultan mucho más convincentes para presentar un fármaco que el propio fabricante) así como también la creación de grupos de consulta. También se puede reunir un grupo de especialistas, y sus conclusiones enviadas a los medios.

\section{REPERCUSIONES}

\section{Sobre la investigación}

La investigación médica constituye una realidad compleja determinada por múltiples factores interdependientes de tipo económico, sociocultural, político y profesional. La escasez de fondos públicos y el interés de algunos investigadores en su autopromoción profesional (el llamado efecto Utah) (41) crean un terreno abonado para que el interés comercial de las empresas farmacéuticas se convierta en uno de los factores más determinante de la investigación actual ya que, como hemos visto antes, por varios motivos la industria se ha convertido en una de sus principales fuentes de financiación y gestión. La primera de las consecuencias de esto es que en las últimas décadas el mayor peso de la investigación recae en la investigación biológica (o biologista) y en los ensayos farmacológicos. En un análisis de Thornley y Adams en 1998 (42) de 2000 ensayos clínicos controlados de intervenciones en la esquizofrenia en 50 años encuentran que el $86 \%$ evaluaban la eficacia de los diferentes fármacos por un $8 \%$ de intervenciones psicoterapéuticas y un $9 \%$ sobre programas de cuidado o tratamiento. Una consecuencia derivada de la anterior es que, al menos en nuestro país, una parte significativa de los clínicos que llevan el peso asistencial y los residentes en formación terminan por creer que la investigación se reduce a la aplicación en un medio hospitalario de una metodología estadístico-correlacional para comprobar hipótesis de contenido biológico y se desaniman a la hora de reivindicar las condiciones necesarias para poder desarrollar otro tipo de investigaciones más holísticas y contextualizadas en nuestro medio, de igual importancia en psiquiatría.

Centrándonos en la investigación en el campo de la biología y farmacológía cabe preguntarse por la influencia de las 
estrategias de marketing-investigación de las multinacionales farmacéuticas que antes hemos descrito. Para psicofarmacólogos como Healy ${ }^{10}$, aunque hayan pasado más de tres décadas, las hipótesis dopaminérgicas sobre la esquizofrenia y las teorías monoaminérgicas sobre la depresión todavía marcan la agenda de la investigación biológica de los trastornos mentales, sin que haya existido una gran avance, aparte de los honores académicos y fondos de investigación que han logrado los neurocientíficos y los psiquiatras (1). Este autor señala cómo actualmente es difícil discernir si algo constituye un verdadero avance en los conocimientos neuroquímicos o es una hipótesis de moda magnificada por intereses comerciales (como ocurrió con la serotonina en la década pasada a la vez que se comercializaban los ISRS).

En cuanto a la investigación psicofarmacológica ya hemos señalado antes cómo las dificultades y los sesgos metodológicos, en parte propiciados por las necesidades de marketing, nos obligan a ser cautelosos a la hora de analizar los resultados sobre los nuevos fármacos. Es interesante señalar cómo ha ido cambiando la nomenclatura de los psicofármacos desde la aparición de la clorpromacina (4). Así dejaron de usarse categorías tradicionales como tranquilizantes mayores o estimulantes que nos hablaban de funciones para pasarse a términos como antidepresivos o antipsicóticos que transmiten la idea de fármacos específicos, para enfermedades o síntomas específicos, una idea que también se extendió en su día con el coma insulínico para la esquizofrenia y el TEC para los trastornos afectivos. Sin embargo, no está tan claro que los psicofármacos sean tratamientos específicos de enfermedades específicas. Este argumento ha ayudado más a la industria a promocionar sus tratamientos y a la psiquiatría a subir de status dentro de la medicina que a los investigadores básicos a formular hipótesis coherentes sobre el funcionamiento de los psicofármacos $(1,4,43)$. Un nuevo ejemplo de cambio de nomenclatura lo tenemos actualmente con el concepto de antipsicótico atípico que reúne a un conjunto heterogéneo de nuevos neurolépticos cuya única característica común podría ser el elevado precio.

La trascendencia de esto es que deja no sólo a los clínicos en una gran incertidumbre, sino también a los gestores y responsables políticos. Los estudios que se realizan son estudios de eficacia y no estudios de eficiencia, generalmente en contextos sanitarios muy diferentes al nuestro como es el norteamericano $(19,42)$. Los estudios farmacoeconómicos, que suelen favorecer a los nuevos fármacos presentan importantes limitaciones como para poder tomar decisiones clínicas y políticas (44). Primero, el método retrospectivo que se usa en la mayoría de los estudios investigados es más propenso a que se seleccionaran los ensayos que originaban mejores resultados, para realizar posteriormente su análisis económico. Segundo, hay una tendencia evidente también en la investigación farmacoeconómica hacia la publicación de estudios con resultados positivos, en detrimento de los que ofrecen resultados negativos. Tercero, se podrían producir influencias indirectas de las 
compañías farmacéuticas hacia los investigadores, dado que éstos pueden recibir emolumentos, directa o indirectamente. Y cuarto, la compañía podría influir en la elaboración de los protocolos o en los criterios de evaluación económica.

\section{Sobre la concepción del trastorno mental}

2.1. Cosificación de la enfermedad mental

La excesiva importancia que se le ha dado a los tratamientos farmacológicos en psiquiatría durante la pasada «década del cerebro» ha propiciado una visión casi hegemónica de la enfermedad mental desde el modelo biomédico. Este modelo se sustenta en una teoría biológica del proceso de enfermar cuyas manifestaciones clínicas aparecen recogidas en las clasificaciones nosológicas categoriales y que precisa de un remedio también de orden biológico (no sólo farmacológico, el TEC también vale) para su tratamiento. Este modelo, de forma inseparable, ha producido una reificación de la enfermedad mental, y que ésta se entienda como una cosa ajena al individuo en cuanto a su origen, mantenimiento y curación. Se trataría en definitiva de un desorden de los neurotransmisores que precisa de una medicación (como si se tratara de un tratamiento hormonal regulador o algo parecido) para su curación. En esta concepción de la enfermedad mental el paciente desempeña un rol pasivo y dependiente de un psiquiatra que oficia de experto «pseudoendocrinólogo». En el encuentro entre ambos el médico tiene que identificar las manifestaciones clínicas del trastorno (independientemente de las circunstancias ambientales o la visión subje- tiva del paciente que pueden confundir y enturbiar el proceso de enumeración de criterios diagnósticos presentes) para prescribir el fármaco más idóneo. Esta visión sustantiva de la enfermedad mental se opone a la óptica histórica, biográfica, de sentido, narrativa imprescindible para intervenir con las personas que sufren los trastornos mentales (45).

\subsection{Repercusión sobre la nosología}

Cuando las empresas farmacéuticas altamente competitivas irrumpieron con los psicofármacos, comenzaron a deformar el sentido del propio diagnóstico psiquiátrico. Como el objetivo es vender más han desarrollado tres estrategia básicas para llevar a cabo este propósito. Aunque se solapan unas con otras, las ejemplificaremos por separado para su mejor entendimiento.

- Nuevos psicofármacos para nuevas enfermedades:

El ejemplo prototipo de esta estrategia de venta es la aparición del alprazolam en el trastorno de pánico. Esta entidad, diferenciada del trastorno de ansiedad, no apareció como tal hasta 1980 con su publicación en el DSM-III. Curiosamente, al año siguiente, con el mercado de las benzodiacepinas ya saturado, se lanzó el alprazolam por la empresa fabricante como un nuevo producto revolucionario que estaba precisamente indicado de forma específica para este trastorno (2). En los años siguientes el trastorno de pánico se convirtió en uno de los trastornos psiquiátricos más prevalentes y el alprazolam en el psicofármaco más vendido. 
- Nuevas indicaciones de enfermedades para los mismos fármacos:

Uno entiende fácilmente que sacar un nuevo psicofármaco al mercado tiene un coste elevado, no tanto en su síntesis como en su promoción para lograr tener aceptación entre consumidores y prescriptores. Mucho más barato y rentable es conseguir que un producto que ya es conocido y tiene un nombre amplíe sus indicaciones y sirva para más cosas de las que fue inicialmente comercializado. Esta estrategia que pudo ser novedosa en un principio es constante ya con todos los productos que salen al mercado. Todos los psicofármacos sirven para varios trastornos $\mathrm{y}$, algunos de ellos, son auténticas panaceas. La paroxetina, sin ir más lejos, está indicada en depresión, trastorno por ansiedad generalizada, trastorno por ansiedad social, trastorno de pánico, trastorno obsesivo compulsivo, trastorno por estrés postraumático y trastornos de la conducta alimentaria. Los partidarios de la psicosis única o la neurosis única pueden aprovechar para reivindicarse pero lo cierto es que las compañías farmacéuticas abogan por lo contrario: crear cada vez más categorías de enfermedades y para todas ellas sirve un mismo psicofármaco, aunque intentan argumentar una supuesta especificidad para cada trastorno.

- Ampliación de los límites de la enfermedad:

$\mathrm{Si}$ bien las anteriores estrategias sirven para que unos psicofármacos tengan más ventas en detrimento de otros, pues teóricamente se dan sólo al grupo de enfermos, esta beneficia a todas las compañías farmacéuticas porque aumenta la demanda, es decir, logra que haya más consumidores (o sea, enfermos). Esta estrategia se sustenta en la influencia sobre el discurso médico y social para poder crear «epidemias» de enfermedades ya conocidas a expensas de incorporar al grupo de enfermos a gente sana. Primero se vende la enfermedad y luego el fármaco.

El diagnóstico de trastorno por déficit de atención-hiperactividad en niños, por ejemplo, se ha incrementado espectacularmente en los últimos años paralelamente a las ventas de Ritalin ${ }^{\circledR}$ en los EE. UU. Las conductas que definen este trastorno se solapan con aquellas que son comunes en niños cuando se sienten frustrados, ansiosos, aburridos, abandonados o estresados de alguna manera. Habría que preguntarse si este fenómeno de prescribir psicofármacos a los niños se debe a un genuino incremento del trastorno o a una estrategia alternativa a la difícil tarea de mejorar la vida familiar y escolar (46).

Otra enfermedad que se ha disparado en los últimos años ha sido la fobia social. Antes del lanzamiento de la moclobemida y de la indicación de la paroxetina, este trastorno apenas era conocido. En los EE.UU. se desarrolló una campaña mediática coordinada por una agencia de relaciones públicas y técnicas comerciales llamada Cohn Wolfe, que trabajaba para la empresa farmacéutica interesada en lanzar su molécula en esta nueva indicación. Los argumentos dirigidos a periódicos, radio y televisión, medios de comunicación e internet afirmaban que este era el tercer diagnóstico psiquiátrico más frecuente en los EE. UU. tras la depresión mayor y la dependencia a alcohol, que 
afectaba a un 13,3\% de la población a lo largo de sus vidas y que el primer y único medicamento aprobado por la FDA para la ansiedad social era el Paxil, (paroxetina) $(46,47)$. Puede parecer muy noble el intentar dar a conocer la enfermedad para que los verdaderamente fóbicos sociales no se sientan excluidos o marcados, pero esta campaña, liderada por una empresa farmacéutica, estaba dirigida principalmente a incrementar las ventas de su producto, y para ello se desvirtuó el diagnóstico, convirtiendo la timidez en una enfermedad psiquiátrica de características epidémicas en los EE. UU.

Pero la mayor epidemia de los últimos años ha sido la depresión. Desde que se comercializó el Prozac «a principios de los 90, han aparecido en el mercado un aluvión de grupos de nuevos antidepresivos (ISRS, IRSN, RIMA, NaSSA, ISRN) con distintas moléculas en cada grupo y varias marcas para cada molécula. Esto creó una competencia tan intensa por conseguir las ventas que al final la mejor estrategia ha sido lograr que gran parte de la población se creyera deprimida. La Defeat Depression Campaign (DDC) desarrollada en 1992 organizada por los colegios oficiales de psiquiatras y médicos generales ingleses y financiada por la industria farmacéutica es un buen ejemplo. Entre las consignas publicitarias destacan que un tercio de la población sufrirá al menos una depresión en su vida y que los antidepresivos se recomiendan para todos aquellos que tengan síntomas moderados o graves (48). Esta popularización de la depresión permite extender los límites de la psiquiatría (en los años 50 la depresión era un tras- torno relativamente raro) y alimenta intereses corporativistas y aumenta el mercado de los psicofármacos. Con esto se ha conseguido que el término depresión se haya vuelto sinónimo de infelicidad, tristeza, estrés, malestar o de cualquier sentimiento desagradable por legítimo que sea. Desde luego se ha contribuido a desestigmatizar este trastorno, pero a costa de que lo padezcamos casi todos.

\section{Sobre los psiquiatras}

El control que ejerce la industria farmacéutica sobre la investigación, la información científica, la formación de los psiquiatras y su incentivación para que prescribamos sus productos nos deja en muy mal lugar. El propósito de las compañías es que tengamos una mentalidad biomédica en el acercamiento al paciente, lo diagnostiquemos (según criterios DSM pero con manga ancha para que se incluyan no pacientes que igualmente se pueden beneficiar de los productos farmacéuticos) y le prescribamos el último prodigio de la psicofarmacología que es de lo más específico para el trastorno en cuestión. Es de suponer que uno no estudia la carrera de medicina, aprueba el MIR y hace la residencia para acabar contando criterios diagnósticos y hacer una receta a continuación, pero una buena parte de los psiquiatras no hace mucho más. Últimamente se habla del queme o burnout de los psiquiatras, pero con esta ampliación de los márgenes de la enfermedad mental parece que hay que tratarlo todo y en un paradigma biologicista que es poco eficaz y reduccionista. 
En mayor o menor medida esto es parte del presente de los psiquiatras y será el futuro de seguir las cosas así. Es muy difícil escapar del pensamiento único omnipresente en cursos, seminarios, revistas, investigaciones y viajes de placer, especialmente para las nuevas generaciones que se incorporan a la profesión con estas estrategias de mercado ya consolidadas.

\section{Sobre la asistencia a los trastornos mentales \\ La implantación de un modelo} biomédico de la enfermedad mental que está calando entre los profesionales y la población general cuyo tratamiento está basado en la administración de psicofármacos cada vez mucho más caros está causando repercusiones graves sobre la asistencia de los enfermos mentales.

Este modelo está propiciando que la oferta asistencial sea la prescripción de psicofármacos como abordaje casi exclusivo de los trastornos mentales. Nadie pone en duda que muchos de los psicofármacos comercializados son útiles para mejorar los síntomas de algunos trastornos, pero también sabemos que la psicoterapia, la rehabilitación, la psicoeducación... son herramientas terapéuticas útiles e imprescindibles por lo que obviarlas produce un perjuicio a nuestros pacientes.

La expansión del mercado de los psicofármacos con la ampliación de los límites de las enfermedades y la popularización de los trastornos mentales está causando la saturación de los dispositivos asistenciales por usuarios sin trastornos mentales que reivindican una asistencia especializada para tratar su malestar. Se ha estimado que alrededor del 20-30\% de los pacientes que acuden a los servicios de salud mental (es decir, en un segundo nivel asistencial) no presentan ningún trastorno mental diagnosticable (49). Como se proporciona más y mejor asistencia a quien más la reclama y no a quien más la necesita según la ley de cuidados inversos, se desvían los recursos de los pacientes graves para atender estas demandas.

Otro perjuicio que ocasiona la industria farmacéutica a nivel asistencial tiene que ver con la viabilidad de la atención sanitaria. El problema actual que está comprometiendo a los sistemas sanitarios europeos públicos basados en la universalidad y la gratuidad de las prestaciones es el creciente gasto que suponen para la economía del país. El mayor problema de la atención sanitaria en este sentido es el gasto farmacéutico que crece exponencialmente año tras año, tanto que obliga a muchos países a replantearse medidas de recorte ${ }^{7}$. Curiosamente, entre los fármacos que más gasto ocasionan al sistema sanitario están los nuevos antidepresivos y los nuevos antipsicóticos.

\section{Sobre el discurso social}

Se puede ganar mucho dinero si se le hace creer a gente sana que está enferma. Para ello, la industria farmacéutica está activamente involucrada en ampliar los límites de la enfermedad tratable y difundir su nueva definición para abrir mercados. A través de los medios de comunicación, como hemos visto, se da publicidad a las enfermedades y se promocionan a consumidores y prescriptores para poder vender los productos que teóricamente las curan. 
Es importante en este sentido ignorar o descalificar abordajes del problema alternativos o el hecho de que el problema tenga una historia natural benigna o autolimitada. Este fenómeno ha conducido a conceptualizar como enfermedad procesos normales (la alopecia), riesgos (osteoporosis, hipercolesterolemia...) o se ha medicalizado el proceso de vivir (nacimiento, sexualidad, envejecimiento y muerte) (6,7). En el campo de la psiquiatría este fenómeno de promocionar enfermedades para vender fármacos con los que «curarlas» ha sido masivo. Sobre el malestar se ha edificado un mercado en el que se ha propiciado el uso cosmético de los psicofármacos y se dan pastillas a los que están tristes (estén o no deprimidos), a los que son tímidos, a los niños revoltosos o al que está nervioso por cualquier problema cotidiano. De esta manera, características omnipresentes de la condición humana se convierten en trastornos psiquiátricos rentables. La enfermedad deja de ser una construcción social y se convierte en una construcción corporativa. Este discurso se ha alimentado de varios mitos: 1.La salud es la ausencia completa de malestar y cualquier sentimiento de este tipo, por legítimo que sea, puede ser considerado como un síntoma de una enfermedad y, por tanto, susceptible de ser tratado como tal. 2.- El aumento de los problemas de salud mental y del número de enfermedades son el resultado de un progreso de la ciencia en cuanto a detección e intervenciones precoces. 3.- Los psicofármacos son eficaces para combatir los sentimientos de malestar. Sin embargo, la ansiedad y la tristeza son respuestas fisiológicas, legíti- mas y adaptativas ante determinados acontecimientos vitales, el aumento de categorías diagnósticas tiene que ver con intereses corporativistas de ciertos lobbies (compañías farmacéuticas, colectivos de profesionales, aseguradoras, grupos de pacientes..) y no existe ningún ensayo clínico que demuestre que los psicofármacos ayuden a resolver más satisfactoriamente los problemas de la vida.

Es curioso cómo, cuando el Valium apareció en los 50, tanto los pacientes como sus médicos deseaban definir sus problemas en términos de ansiedad una vez que existía un fármaco efectivo para tratarla. En los 90, con la irrupción del Prozac, , se cambió el discurso y el malestar o el estrés a partir de entonces se describe como depresión. La creación de este mercado del malestar y su difusión en la población general ha provocado una psiquiatrización de la vida cotidiana. Ahora las desgracias tienen una solución química y se crean expectativas de felicidad y bienestar mediante el consumo de psicofármacos. Esta medicalización del malestar produce una expropiación individual y comunitaria de los sentimientos de los ciudadanos (que ya no pueden controlarlos solos y precisan de una pastilla que lo haga por ellos) y al otorgar a la conducta, pensamientos y emociones determinantes biológicos, colocamos al individuo en el lugar de un espectador pasivo y enfermo ante los avatares de su vida. En este sentido, dar pastillas contra el malestar supone sancionar como individuales problemas que pueden tener un sustrato social (y se propugna la adaptación personal frente a situaciones sociales injustas) y significa res- 
paldar el debilitamiento de las redes tradicionales de contención y aceptar las imposiciones que el mercado ordena.

\section{Conclusiones}

1.- La industria farmacéutica se ha convertido en las últimas décadas en una de las actividades económicas más lucrativas cuyos enormes beneficios en psiquiatría dependen en mayor medida del desarrollo y perfeccionamiento del marketing que de verdaderos avances científicos.

2.- Las estrategias de mercado pueden terminar por secuestrar el pensamiento psiquiátrico debido a su enorme poder de influencia sobre la investigación, la información científica, la formación y los incentivos de los profesionales.

3.- Parte de la profesión psiquiátrica es conscientemente cómplice de este secuestro mientras que otra parte sufre en mayor o menor medida un síndro- me de Estocolmo que le impide valorar con independencia la influencia de los intereses comerciales en la psiquiatría.

4.- La falta de inversiones públicas en investigación y formación favorece la posición dominante de la multinacionales farmacéuticas en estos aspectos tan claves de un sistema sanitario público.

5.- Los intereses comerciales de las compañías farmacéuticas tienen una gran repercusión en el discurso social, ya que contribuyen a la psicofarmacologización inútil de malestares culturales y sociales que incrementan continuamente el gasto farmacéutico, y amenazan la viabilidad de los sistemas nacionales de salud.

6.- Psiquiatras, asociaciones profesionales y la propia industria deberían reflexionar sobre este fenómeno que puede llevar a la psiquiatría a morir de éxito.

\section{TABLAS}

Tabla I. Clases terapéuticas líderes en ventas farmacéuticas 2001

\begin{tabular}{|c|l|c|c|c|}
\hline Ranking & $\begin{array}{l}\text { Case terapeútica auditada } \\
\text { mundialmente }\end{array}$ & $\begin{array}{c}\text { Ventas en 2001 } \\
\text { (millones de dólares) }\end{array}$ & \% ventas globales & \% variación anual \\
\hline 1 & Antiulcerosos & 19.500 & $6 \%$ & $+14 \%$ \\
\hline 2 & Hipolipemiantes & 18.900 & 5 & +22 \\
\hline 3 & Antidepresivos & 15.900 & $\mathbf{5}$ & +16 \\
\hline 4 & AlNES & 10.900 & 3 & +4 \\
\hline 5 & Antagonistas del calcio & 9.900 & 3 & +30 \\
\hline 6 & Antipsicóticos & 7.700 & 2 & +30 \\
\hline 7 & Antidiabéticos orales & 7.600 & 2 & +5 \\
\hline 8 & Inhibidores ACE & 7.500 & 2 & 0 \\
\hline 9 & Cefalosporinas y comb. & 6.700 & 2 & +22 \\
\hline 10 & Antihistamínicos & 6.700 & $32,0 \%$ & $+16 \%$ \\
\hline Total 10 & Primeras clases terapéuticas & $\$ 111.300$ & & 2 \\
\hline
\end{tabular}


Tabla II. Productos líderes en ventas año 2000.

\begin{tabular}{|c|l|c|c|c|}
\hline Ranking & $\begin{array}{l}\text { Ventas de productos } \\
\text { auditados mundialmente }\end{array}$ & $\begin{array}{c}\text { Ventas año 2000 } \\
\text { (millones de dólares) }\end{array}$ & \% ventas globales & \% variación anual \\
\hline 1 & Lipitor® & 6.100 & $1,9 \%$ & $+9 \%$ \\
\hline 2 & Losec/Prilosec $\AA$ & 5.400 & 1,7 & $+44 \%$ \\
\hline 3 & Zocor & 4.400 & 1,4 & $+15 \%$ \\
\hline 4 & Norvasc & 3.300 & 1,1 & $+15 \%$ \\
\hline 5 & Ogastro/Prevacid & 3.100 & 1,0 & $+33 \%$ \\
\hline 6 & Prozac & 2.900 & 0,9 & $-1 \%$ \\
\hline 7 & Seroxat/Paxil & 2.400 & 0,8 & $+20 \%$ \\
\hline 8 & Zyprexa & 2.400 & 0,8 & $+30 \%$ \\
\hline 9 & Celebrex & 2.400 & 0,7 & $+65 \%$ \\
\hline 10 & Zoloft (sertralina) & 2.200 & 0,7 & $+12 \%$ \\
\hline & Total 10 productos líderes en ventas & $\$ 34.500$ & $10,9 \%$ & $+21 \%$ \\
\hline
\end{tabular}

\section{NOTAS}

1. Estas cifras son difíciles de imaginar para los ciudadanos comunes. Silva pone las siguientes comparaciones para entender la magnitud de cifras: con los 18,9 Billones de dólares de ventas sólo en Latinoamérica, podríamos solucionar el hambre y la desnutrición en el mundo (9 Billones de dólares), eliminar el analfabetismo mundial (5 Billones de dólares), y poner en marcha un programa global de asistencia a todos los pacientes con HIV de Sudáfrica

2. A pesar de estos ingentes beneficios las empresas farmacéuticas se han visto envueltas en polémicas de gran significación social y ética, como por ejemplo su posición en el precio y las patentes de los medicamentos contra el SIDA para los países del tercer mundo (investigaciones que también habían contado con fondos públicos).

3. Hay que tener en cuenta que en estas cifras se incluyen los gastos de moléculas similares que no han llegado a la fase de registro y los costes de marketing (tanto en la fase prelanzamiento como en la fase de promoción)

4. En el año 2000 la compañía Merck gastó 161 millones de \$ en la publicidad de un AINE, más de lo que gastaron compañias como Pepsico (125 millones) o Budweiser (146 millones).

5. Véase por ejemplo: Memoirs of methods used to sell drugs. MaLAM. March/April 1999 Vol 17 N 3/ 4 ISSN 1321-571X . En este articulo, un antiguo empleado del departamento médico de una compañía farmacéutica cuenta los métodos que él observó en tres de las empresas en las que trabajo para vender los productos. Se puede consultar en la edición electrónica de MaLAM (http://healthyskepticism.org/editions/IN9903.htm)

6. Además las multinacionales no airean las ventajas fiscales y de financiación pública de que ya gozan para I+D. En EE UU, que acapara el $40 \%$ de las ventas mundiales, la iniciativa privada contribuyó en un $52 \%$ al conjunto de la I+D de sanidad, pero la oficial, los Institutos Nacionales de Salud, aportó otro 30\%: 45 de los 50 fármacos más vendidos en los años noventa recibieron dinero público En 1995 el Massachussets Institute of Tecnology (MIT) de Boston descubrió que de los 14 medicamentos más prometedores para el último cuarto de siglo 11 tenían su origen en trabajos financiados por el estado.(The New York Times on the web, 23 de Abril de 2000

7. Una extensa bibliografía sobre las relaciones entre la industria y medicina se puede encontrar en http:// www.healthyskepticism.org/bibliogr.htm

8. Richard Horton, editor de The Lancet, ha declarado al diario inglés The Independent: «Estamos hartos de ser manipulados por la industria farmacéutica. El $90 \%$ de los estudios científicos sobre nuevos fármacos que llegan a nuestra redacción están tan manipulados a favor del producto que no pueden ser publicados sin una revisión previa. Las investigaciones se usan más como ejercicio de mercadotecnia que como trabajo científico». 


\section{DEBATES}

9. Un buen ejemplo de esto es cómo de un sólo estudio con olanzapina han aparecido publicados más de 80 artículos19.

10. David T. Healy psicofarmacólogo del Departamento Académico de Psicología Medica del North Wales Hospital fue rechazado para un puesto en la Universidad de Toronto que le había sido prometido tras pronunciar una conferencia («Psychopharmacology \& the government of the self»). Se ha sugerido que este rechazo se debió a las presiones de la industria en los medios académicos. Para ver el texto íntegro de la conferencia y los documentos sobre la polémica se puede consultar en http://www.pharmapolitics.com/index.htlm

\section{BIBLIOGRAFÍA}

1. Healy D. The psychopharmacological era: notes towards a history. Journal of psychopharmacology 1990; 4(3): 152-167

2. Shorther E. A history of Psychiatry.(1997) John \& Wiley Inc. Ed. 1997. (Traducción española en J\&C Ediciones Médicas S.L1999, Barcelona ).

3. Sachs RM, Dieck GS. (1989) A view from industry. En Strom BL. Pharmacoepidemiology. Churchill Livinstone, New York.

4. Moncrieff J. An investigation into the precedents of modern drug treatment in psychiatry. History of Psychiatry 1999; 10: 475-490.

5. Fernández-Liria A. Gestión del malestar y sociedad espectacular-mercantil. Sin publicar.

6. Moynihan R, Smith R. Too much medicine? (editorial). BMJ 2002; 324:859-860.

7. Moynihan R, Heath I, Henry D. Selling sickness: the pharmaceutical industry and disease mongering. BMJ 2002; 324: 886-891.

8. O’Reilly B.There's still gold in them thar pills. Fortune magazine, Lunes 23 de Julio de 2001

9. Gury M. IMS reports 12 percent growth in 2001 audited global pharmaceuticals sales. IMS Health 2002 http://www.ims-global.com/insight/news_story/0103/ news_story_010314.htm

10. Silva CD. Introducción al Seminario: Proyecto Goliat: La industria farmacéutica y su impacto en la prescripción de fármacos. En: http://www.edupsi.com/goliat.htm

11. Scholdelmayer WS. Public citizen update: competition and pricing Iisues in the pharmaceutical Market. Prime Institute. University of Minnesota, 2000.

12. Soger A, Socolar D. Pharmaceutical marketing and research spending: the evidence does not support PhRMA's claims. Presentado en: American Public Health Association Annual Meeting, 2001, Atlatanta, Gerogia. Se puede encontrar en http::// dcc2.bumc.bu.edu/hs/ushealthreform.htm

13. Prescription drugs and Mass Media adversiting. NIHCM,2001. http://www.nichm.org/ DTCbcie/2001.pdf

14. Bodenheimer T.Uneasy alliance: clínica investigators and the pharmaceutical industry. N Engl J Med 2000; 342: 1539-1544 
15. Esteve Soler J. La industria farmacéutica como la impulsora de la I+D en el sector sanitario. Revista de la administración sanitaria 1998; 11(7):1-9

16. Ferrán Mercadé M. La financiación de las sociedades científicas y la industria farmacéutica. Atención Primaria 2002;29:327-328

17. Freemantle N Anderson IM, Young P. Predictive value of pharmacological activity for the relative efficacy of antidepressant illness: a randomized double blind controlled trial. Br J Psychiatry 2000; 177:292-302.

18. Moncrieff J. Are antidepressants overrated? A review of metodological problems in antidepressant trials. J Nerv Ment Dis 2001; 189: 288-295

19. Mata Ruiz I, Ortiz lobo A. Sesgos y dificultades en la investigación de los nuevos antipsicóticos. Rev Asoc Esp Neuropsiq; 2001, 79: 57-74.

20. Smith R. Maintaining the integrity of the scientific record (editorial). BMJ 2001; 323:588

21. Sponsorship, authorship, and accountability (editorial). The Lancet 2001; 358: 854856.

22. Just how tainted has medicine become? (editorial). The Lancet 2002; 359:

23. Fernández Liria A. La nueva desmedicalización de la Psiquiatría. Psiquiatría Pública 1998; 10(2): 81-82.

24. Scandal of scientists who take money for papers ghostwritten by drug companies. The Guardian 7 de Febrero 2002.

25. Gilbody SM, Song F, Eastwood AJ, Sutton A. The causes, consequences and detection of publication bias in psychiatry. Acta Psychiatr Scand 2000;102: 241.249.

26. Couhdry NK, Stelfox HT, Detsky AS. Relations between Authors of clinical practice guidelines and the pramaceutical industry. JAMA 2002; 287:612-617

27. Sheldon TA, Smith GD. Consensus conferences as drug promotion. The Lancet 1993; 334: 100-102.

28. Interacctions with the pharmacuetical Industry: experiences and attitudes of psychiatric residents, interns and clerks. CMAJ, 1995; 153:553

29. Wazana A. Physicians and the pharmaceutical industry. Is a gift ever just a gift? JAMA 2000; 283: 373-380

30. Peay MY, Peay ER. The role of commercial sources in the adoption of a new drug. Soc Sci Med 1988; 26: 1183-1189.

31. Lexchin J. Interactions between physicians and pharmaceutical industry. CMAJ 1993; 149: 1401-1407.)

32. Haayer F. Rational prescribing and sources of information. Soc Sci Med 1982; 16: 2017-2023

33. Wolfe SM. Why do American drug companies spend more than $\$ 12$ billion a year pushing drugs? Is it education or promotion? J Gen Intern Med 1996; 11: 637-639

34. Randall T. Kennedy hearings say no more free lunch — or much else - from drug firms. JAMA 1991; 265: 440-442 
DEBATES

35. Coyle SL. Physician-Industry relations. Part 1: individual physicians. Annals of Internal Medicine 2002; 136(5): 396-402.

36. Komesaroff PA, Kerridge IH. Ethical issues concerning the relations between medical practitiones and the phramaceutical industry. The medical journal of Australia 2002; 176(3): 118-121

37. Memoirs of methods used to sell drugs. MaLAM 1999; March/April Vol 17 No 3/ 4. http://www.healthyskepticism.org/editions/IN9903.htm

38. Coyle SL. Physician-Industry relations. Part 2: Organizational issues. Annals of Internal Medicine 2002; 136(5): 403-406.

39. Bowman MA. The impact of drug company funding on the content of continuing medical education. J Contin Educ Health Prof 1986; 6:66

40. Bowman MA, Pearle DL. Changes in drug prescribing patterns related to commercial company funding of continuing medical education. J Contin Educ Health Prof., 1988; $8: 13-20)$

41. Tizón JL. Investigación y docencia en salus mental (I). Reflexiones del clínico antes de la autoinmolación. Rev Asoc Esp Neuropsiq 2001; 77: 69-96

42. Thornley B, Adams C. Content and quality of 2000 controlled trials in schizophrenia over 50 years. BMJ 1998; 317: 1181-1184

43. Van Praag HM. Inflationary tendencies in judging the yield of depresión research. Neuropsychobiology 1998; 37(3):130-141

44. Friedberg M, Saffran B, Stinson T, Nelson W, Bennett C. Evaluation of conflict of interest in economic analyses of new drugs used in oncology. JAMA 1999; 282 (15): 1453-1457

45. Fernández Liria A. Psiquiatría y psicofármacos en el nuevo siglo. Boletín de la AMSM 1999; (invierno):18-25

46. Double D. The limits of psychiatry BMJ 2002;324:900-904

47. El País. Así se vendió la «píldora de la timidez»

48. Moncrieff J Psychiatric imperialism: the medicalisation of modern living. http:// www.critpsynet.freeuk.com/sound.htm

49. Ortiz Lobo A, García Moratalla B, Mata Ruiz I. Las consultas en un CSM de personas sin trastorno mental. Ponencia VI Congreso de la AMSM (AEN) Madrid 6,7 junio 2002.

* Psiquiatras. Centro de Salud Mental de Salamanca. Área 2. Madrid. 